Results Correlations between patients' and physicians' assessments were higher for SLAM-nolab: SWE-SLAQr total, $\rho=0.69$, Symptom score, $\rho=0.67$, and Patients global, $\rho=0.68$ than for SLAM: SWE-SLAQr total, $\rho=0.51$, Symptom score, $\rho=0.49$, and Patients global, $\rho=0.53$. The items fatigue $(\rho=0.72)$ and alopecia $(\rho=0.71)$ showed highest degree of correlation, and dyspnea/ pleuritic chest pain had the lowest correlation between patients' and physicians' assessments $(\rho=0.19, \mathrm{p}=0.039)$. Correlations with SLEDAI-nolab were lower $(\rho \leq 0.36)$ for all subscales. No correlations were found between patients' and physicians' assessments when using SLEDAI-2K $(\rho<0.09$ for all).

Conclusions We conclude that SWE-SLAQr performed equally well as SLAQ, demonstrating that the shorter version can be used to monitor disease impact. We encourage further use of SWE-SLAQr and recommend its implementation in clinical care, we believe it is especially well suited to support digital and telephone contacts. However further attention is needed to evaluate the discrepancy between physicians' and patients' evaluation of thoracic pain/symptoms.

\section{P191 CHINESE SYSTEMIC LUPUS ERYTHEMATOSUS TREATMENT AND RESEARCH GROUP (CSTAR) REGISTRY - SUBJECTIVE WELL-BEING IN PATIENTS WITH SYSTEMIC LUPUS ERYTHEMATOSUS}

\begin{abstract}
${ }^{1}$ Yue Shi, ${ }^{2,3}$ Dandan Bi, ${ }^{4}$ Yanhong Wang, ${ }^{5}$ Ruofan Li, ${ }^{6}$ Lijun Wu, ${ }^{7}$ Cheng Zhao, ${ }^{8}$ Zhenbiao $\mathrm{Wu},{ }^{9}$ Xinwang Duan, ${ }^{10}$ Jian $\mathrm{Xu},{ }^{11}$ Feng Zhan, ${ }^{12}$ Min Yang, ${ }^{13}$ Shengyun Liu, ${ }^{14}$ Qin Li, 'Shuo Zhang, 'Lingshan Liu, 'Jiuliang Zhao, 'Xinping Tian, ${ }^{2,3}$ Xinying Li, ${ }^{1}$ Qian Wang, 'Xiaofeng Zeng, CSTAR Co-authors. 'Dept. of Rheumatology, Peking Union Medical College Hospital, Peking Union Medical College and Chinese Academy of Medical Sciences, National Clinical Research Center for Immunologic Diseases, Ministry of Science and Technology, Key Laboratory of Rheumatology and Clinical Immunology, Ministry of Education, Beijing; ${ }^{2}$ CAS Key Laboratory of Mental Health, Institute of Psychology, Beijing; ${ }^{3}$ University of Chinese Academy of Sciences, Beijing; ${ }^{4}$ Dept. of Epidemiology and Biostatistics, Institute of Basic Medical Sciences Chinese Academy of Medical Sciences, School of Basic Medicine Peking Union Medical College, Beijing; ${ }^{5}$ Dept. of International Education, The Experimental High School Attached to Beijing Normal University, Beijing; ${ }^{6}$ Dept. of Rheumatology, The People's Hospital of Xinjiang Autonomous, Urumqi; ${ }^{7}$ Dept. of Rheumatology, First Affiliated Hospital of Guangxi Medical University, Nanning; ${ }^{8}$ Dept. of Clinical Immunology and Rheumatology, Xijing Hospital Affiliated with The Fourth Military Medical University, Xi'An; ${ }^{9}$ Dept. of Rheumatology, The Second Affiliated Hospital of Nanchang University, Nanchang; ${ }^{10}$ Dept. of Rheumatology, First Affiliated Hospital of Kunming Medical University, Kunming; ${ }^{11}$ Dept. of Rheumatology, Hainan Provincial People's Hospital, Haikou; ${ }^{12}$ Dept. of Rheumatology, Nanfang Hospital, Southern Medical University, Guangzhou; ${ }^{13}$ Dept. of Rheumatology, First Affiliated Hospital of Zhengzhou University, Zhengzhou; ${ }^{14}$ Dept. of Rheumatology, The First People's Hospital of Yunnan Province, Kunming, China
\end{abstract}

10.1136/lupus-2020-eurolupus.233

Objectives Systemic lupus erythematosus (SLE) can significantly influence patients' quality of life and subjective wellbeing (SWB). This study compared SWB statuses in SLE patients and analysed their relationship with clinical manifestations, emotional variables and related positive factors.

Methods Overall, 1779 SLE patients from the Chinese SLE Treatment and Research Group (CSTAR) and 203 age and gender-matched individuals from the general population without self-reported SLE were invited to complete measures of SWB, emotional variables and related positive factors. These patients' clinical data in CSTAR were also linked to investigate the influence of clinical manifestations and psychological factors on SWB among SLE patients.

Results SWB, self-efficacy and resilience were significantly lower $(\mathrm{P}<0.001)$ in SLE patients than in the general population, and self-esteem, depression and anxiety were significantly higher $(\mathrm{P}<0.001)$. Among the SLE patients, life satisfaction was significantly positively correlated with patients' age $(\mathrm{P}<0.001)$ and the age at diagnosis $(\mathrm{P}<0.001)$ and negatively correlated with SLE disease activity index (SLEDAI) $(\mathrm{P}<0.05)$. Premature gonadal failure $(\mathrm{PGF}) \quad(\mathrm{P}<0.05)$ was significantly associated with positive and negative affect scores. In active SLE patients with $\operatorname{SLEDAI} \geq 4$, depression and resilience were life satisfaction predictors. However, anxiety, selfesteem and resilience predicted life satisfaction changes in in patients with SLEDAI $<4$.

Conclusion SWB provides useful insight into the impact of SLE on mental health and opportunities to improve quality of life and clinical care. PGF and disease activity emerge as are predictors of SWB status and potential therapeutic targets to develop positive attitude in SLE patients.

\section{P192 EVALUATION OF PREDICTIVE FACTORS OF WORSE PROGNOSIS IN LUPUS NEPHRITIS: FOCUS ON NEW PATHOGENETIC PATHWAYS}

${ }^{1}$ Valentina Varriano, ${ }^{1}$ Annamaria Paglionico, ${ }^{2}$ Luca Petricca, ${ }^{1}$ Clara Di Mario, ${ }^{2}$ Maria Rita Gigante, ${ }^{1}$ Giacomo Tanti, ${ }^{2}$ Barbara Tolusso, 1,2Elisa Gremese. 'Institute of Rheumatology, Università Cattolica del Sacro Cuore, Rome, Italy; ${ }^{2}$ Division of Rheumatology, Fondazione Policlinico Universitario 'A. Gemelli'- I.R.C.C.S., Rome

\subsection{6/lupus-2020-eurolupus.234}

Purpose To evaluate the prognostic factors in a cohort of patients with lupus nephritis (LN) focusing on of the IL-17, 23 axis as new pathogenetic pathway.

Patients and Methods $91 \mathrm{LN}$ patients were enrolled. Laboratory, immunological and disease activity data were collected at the baseline and at 6(T6),12(T12),24(T24) months and at the last follow-up(FU).84 renal biopsies were evaluated according to ISN/RPS classification, assessing the inflammatory interstitial infiltrate using the BANFF score. Baseline serum levels of IL17 and IL-23 were assessed by ELISA in 37 patients.

Results Among the 84 renal biopsies evaluated $77 \%$ belonged to class III and IV; $41,8 \%$ of patients had an interstitial infiltrate $<5 \%, 35.2 \%$ between $5 \%$ and $25 \%$ and $15,4 \%$ above $25 \%$. Regarding immunological data $35,2 \%$ of patients revealed a seropositivity for antiphospholipid antibodies (APL $+)$. Serum level of IL-17 and IL-23 were $0.12 \pm 0.15 \mathrm{pg} / \mathrm{ml}$ and $27.7 \pm 9.12 \mathrm{pg} / \mathrm{ml}$ respectively. Through the ROC curves analysis we found a cut off value of $25.89 \mathrm{pg} / \mathrm{ml}$ of IL-23 for remission at T6. Among the 10 patients with a IL-23 level above this cut-off none achieved remission at T6 and at the univariate analysis a serum level of IL-23 above the cut-off was associated with an interstitial infiltrate $>5 \%$ at renal biopsy and persistent proteinuria. Finally, we conducted an univariate and multivariate analysis for each renal outcome considered. We found that an inflammatory interstitial infiltrate $>5 \%$ and APL+ were associated with worse renal outcome in terms of early and persistent remission, chronic damage, persistent proteinuria, and renal flare both in univariate and multivariate analysis. Higher serum level of IL-23 was associated with persistent proteinuria, renal flare and tended to be associated to chronic renal damage.

Conclusion Interstitial infiltrate and APL + resulted as the strongest predictors of worse renal outcome. An higher serum level of IL-23 resulted as a negative prognostic factor highlighting its possible role as a biomarkers of more aggressive disease. 